\title{
Postcoincidence trajectory duration affects motion event perception
}

\author{
KATSUMI WATANABE and SHINSUKE SHIMOJO \\ California Institute of Technology, Pasadena, California
}

\begin{abstract}
In a two-dimensional display, identical visual targets moving toward and across each other with equal, constant speed can be perceived either to reverse their motion directions at the coincidence point (bouncing percept) or to stream through one another (streaming percept). Although there is a strong tendency to perceive the streaming percept, various factors have been reported to induce the bouncing percept, such as a sound or a visual flash at the moment of the visual target coincidence. By changing duration of the postcoincidence trajectory (PCT), we investigated how long it would take for such bounce-inducing factors to be maximally effective after the visual coincidence. With bounceinducing factors, the percentage of the bouncing percept did not reach its maximal level immediately after the coincidence but increased as a function of PCT duration up to 150-200 msec. The results clearly reject the possibility of the cognitive-bias hypothesis about the bounce-inducing effect and suggest rather that the bounce-inducing factors have to interact with the PCT for some period after the coincidence to be maximally effective.
\end{abstract}

Consider two identical visual targets moving toward each other with equal and constant speed in a twodimensional display. They eventually coincide and move apart. Due to the two-dimensional nature of the display and the correspondence problem (e.g., Cavanagh, Arguin, \& von Grunau, 1989; Ramachandran \& Anstis, 1983; Ullman, 1979), two types of motion event perception are possible but mutually exclusive: the targets reversing their motion directions at the coincidence point or the targets moving in the same direction (e.g., Johansson, 1950; Metzger, 1934; Michotte, 1946/1963). Since most observers describe the reversal of motion direction as "bouncing" and the nonreversal event as "streaming," we will call them the bouncing percept and the streaming percept, respectively.

Because of its motion-related ambiguity, the streaming/ bouncing motion configuration has been investigated mostly with respect to visual motion integration (Bertenthal, Banton, \& Bradbury, 1993; Bertenthal \& Kramer, 1988; Goldberg \& Pomerantz, 1982; Gorea \& Labarre, 1997a, 1997b; A. B. Sekuler \& R. Sekuler, 1999; R. Sekuler, A. B. Sekuler, \& Brackett, 1995; Watanabe \& Shimojo, 1998). Studies generally agree that the streaming percept is dominant, though not exclusively, when subjects just look at this motion display. This streaming dominance makes sense because the display may contain several visual cues favoring the streaming event. In the natural environment, two solid objects never occupy the same loca-

This work was supported by the Sloan Foundation for Theoretical Neurobiology to K.W. The authors thank Sverker Runeson, Leo Poom, and anonymous reviewers for useful comments and suggestions for an earlier version of the manuscript and Johanna Weber for valuable comments and editing. Correspondence should be addressed to K. Watanabe, California Institute of Technology, Computation and Neural Systems, Mail Code 139-74, Pasadena, CA 91125 (e-mail: kw@ caltech.edu). tion at the same time. When two objects bounce off each other, they reverse their motion direction as soon as their visual contours contact each other. Therefore, the spatialtemporal properties of the physical bouncing event at the critical moments of coincidence would differ from the streaming/bouncing display described above.

Nonetheless, subjects occasionally report the bouncing percept with the streaming/bouncing motion display, which suggests that the display is in fact ambiguous. Furthermore, various factors have been reported to induce perception of bouncing, such as a transient visual distractor (Watanabe \& Shimojo, 1998), a transient sound (R. Sekuler, A. B. Sekuler, \& Lau, 1997), and a momentary pause of the moving targets (Bertenthal et al., 1993) at the moment of the visual target coincidence.

The streaming/bouncing motion configuration provides us with a unique chance to investigate how the visual system integrates information from different sources in order to derive a certain perceptual outcome. Our preliminary observations suggested that the duration of the target trajectory after the visual coincidence (postcoincidence trajectory, PCT) might affect the likelihood of streaming versus bouncing percept.

How could the duration of the PCT after the visual coincidence affect the motion event perception at the moment of the coincidence? It clearly indicates the presence of an effective time window within which the bounce-inducing factors interact with mechanisms for motion event perception after the target coincidence. If this is true, it would be inconsistent with the cognitive-biashypothesis about the bounce-inducing effect-namely, the hypothesis that the bounce-inducingeffect may occur simply because the presence of a transient visual or auditory stimulus resembles the physical collision of two objects and, therefore, subjects might explicitly infer the reversal of motion direc- 


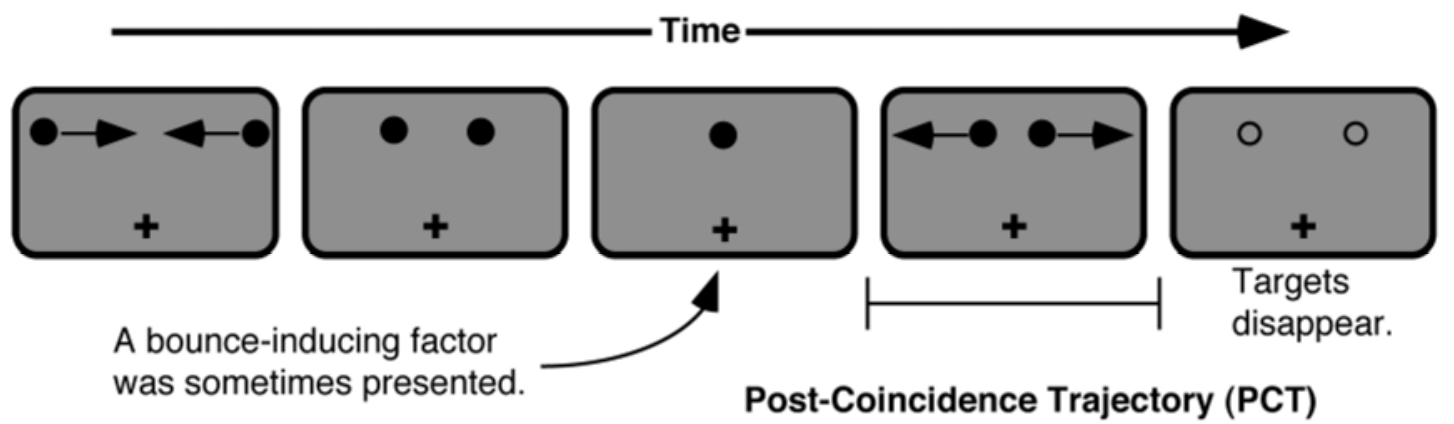

\begin{abstract}
Figure 1. The stimulus configuration. The two black disks appeared, immediately started to move, coincided, continued to move, and disappeared after a randomly assigned interval. At the moment of target coincidence, a bounceinducing factor was introduced (sound in Experiments 1-3, a visual flash of a ring or a momentary pause of the targets in Experiments 4-6), except in Experiment 3 in which the sound was delivered 100 msec before or after the visual coincidence.
\end{abstract}

tion from the presence of these bounce-inducing factors. The cognitive-bias hypothesis predicts that there should not be a PCT-duration dependency.

In the present study, we conducted six experiments to confirm and extend our preliminary observations. Specifically, we were interested in how the development of the bouncing percept, induced by various factors, would depend on the duration of the PCT. For this purpose, we systematically varied the PCT duration along with the established bounce-inducing factors and asked subjects to report their percept: streaming, bouncing, or unsure.

\section{EXPERIMENT 1}

The question we examined in Experiment 1 was the effect of a transient sound (R. Sekuler et al., 1997) in our PCT paradigm. We intended to find when or at what point in the PCT the sound begins biasing perception toward bouncing and when this bias would reach the maximum level. The PCT duration might have no influence on the bounce-inducing effect of the sound. That is, after the visual target coincidence with the presentation of the sound, the dominant streaming percept may switch to the bouncing percept without delay. This outcome would argue in favor of the cognitive-bias hypothesis of the bounceinducing effect. Alternatively, the percentage of the bouncing percept might increase systematically as a function of the PCT duration. That is, for the sound to induce the bouncing percept, some period of interactions between the sound and information from the PCT may be necessary.

\section{Method}

Subjects. Nine people ( 5 females and 4 males, including the authors) participated as subjects in the experiment. Except for the authors, all were naive as to the purposes and hypotheses motivating the study. Their ages ranged from 20 to 43 years. They had normal or corrected-to-normal vision and hearing and reported no severe difficulty with the tasks. They were treated in accordance with the ethical guidelines of the American Psychological Association (1992).
Stimuli. Visual stimuli were displayed on a Sony color monitor, controlled by a Silicon Graphics Indigo 2 workstation, in a dark room. The refresh rate of the monitor was $60 \mathrm{~Hz}$, but the actual frame rate of the visual display was $20 \mathrm{~Hz}$. This rather slow frame rate was used to ensure synchrony between visual and auditory stimuli. With these visual display settings, we did not observe phenomenal duplication phenomena (i.e., a moving spot appears as two or more separate spots moving together). Also, a preliminary observation indicated that the frame rate, ranging from 20 to $75 \mathrm{~Hz}$, did not affect the results substantially.

A black fixation cross $\left(0.01 \mathrm{~cd} / \mathrm{m}^{2}, 0.35^{\circ}\right.$ in visual angle $)$ and a black frame $\left(3.9^{\circ}\right.$ in height and width) were displayed at the center of the monitor throughout each session. The background was gray with a luminance value of $8.5 \mathrm{~cd} / \mathrm{m}^{2}$. At the beginning of each trial, two black disks $\left(0.16^{\circ}\right.$ in diameter $)$ were presented near the opposite edges of the frame. The disks were initially separated about $3.27^{\circ}$ and placed $1.47^{\circ}$ above the fixation cross. The disks moved horizontally toward each other and, about $1 \mathrm{sec}$ after, coincided above the fixation cross (Figure 1). They continued to move but disappeared after various time intervals, which ranged from 0 to $350 \mathrm{msec}$ (from 0 to 7 frames). The frame-to-frame spatial offset of the targets was about $0.08^{\circ}$, which resulted in a constant speed of $1.64^{\circ}$ per second. In half of the trials, when the two disks were superimposed, a single sound was presented through the computer's built-in speaker (located about $10^{\circ}$ below the fixation cross). The sound was a digitized $1.8 \mathrm{-kHz}$ sound with a duration of $3 \mathrm{msec}$. It had a virtually zero rising time and an approximately $1.5-\mathrm{msec}$ falling time. The highest sound pressure level was $58 \mathrm{~dB}(\mathrm{~A})$ at the subject's ear. There was background noise of about $53 \mathrm{~dB}(\mathrm{~A})$.

Procedure. The subject observed the stimulus display binocularly and binaurally from a distance of $80 \mathrm{~cm}$ while fixating at the fixation cross throughout each trial. The two disks appeared, immediately started to move, coincided, continued to move, and disappeared after a randomly assigned interval. The subject was asked to report whether the two disks appeared to "bounce off" (reversing their directions of motion) or "stream through" (no reversal of motion direction) each other by choosing and pressing one of the mouse buttons. If the subject was unsure about the percept, he/she could press the third mouse button (three-alternative, forced choice). However, the subject was instructed to minimize the number of unsure responses. Immediately after the subject's response, another trial began. There were eight different PCT durations $(0,50,100$, $150,200,250,300$, and $350 \mathrm{msec}$ ) and two sound conditions (nosound or sound). For each combination of these factors, 20 trials 


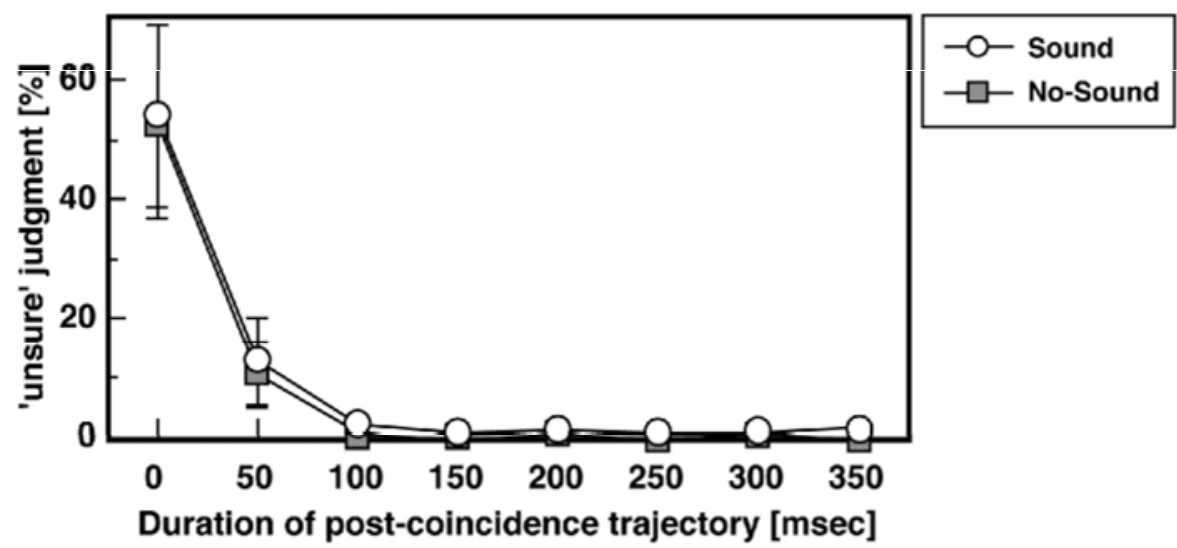

Figure 2. The effect of the PCT duration on the percentage of "unsure" responses in Experiment 1 . The vertical axis shows the averaged percentage of "unsure" responses $(N=9)$, with $T$ bars indicating $\pm 1 S E$. Except for in the 0-msec PCT condition, the subjects were quite sure about their judgments. This was true for all the other experiments in the present study.

were repeated (the method of constant stimuli; PCT duration [8] $\times$ sound condition $[2] \times$ repeat $[20]=320$ trials). A full experiment was divided into four sessions. Each session consisted of 80 trials.

\section{Results}

The pooled mean percentages of "unsure" responses in both stimulus conditions were about $53 \%$ for the 0 msec PCT condition, $11 \%$ for the $50-\mathrm{msec}$ PCT condition, and $1 \%$ for the other PCT duration conditions (Figure 2). A two-way analysis of variance (ANOVA) for the percentage of "unsure" responses showed no significant difference between the no-sound and sound conditions $[F(1,8)=0.122, p=.728]$, whereas it showed the main effect of PCT $[F(7,56)=19.09, p<.001]$. Except for when there was no PCT, the percentage of "unsure" responses was negligible compared with those of streaming and bouncing responses.

Figure 3 shows the averaged percentage of bouncing responses across the 9 subjects as a function of the PCT duration. In order to see trends in the dependency of streaming/bouncing perception on the PCT duration, we approximated the data with linear, logarithmic, and logistic functions and chose the best fit for each sound condition. The no-sound condition was approximated best, though poorly, with a logarithmic function $\left(R^{2}=.694\right)$, and the sound condition was fitted best with a logistic function $\left(R^{2}=.971\right)$.

The 0 -msec PCT condition was excluded from the following statistical analyses, since it would compromise the analyses due to the high percentage of "unsure" responses. A two-way ANOVA for the percentage of "bouncing" responses was performed on the variables of PCT duration and sound condition. There were main effects of both PCT duration $[F(6,48)=3.73, p<.005]$ and sound condition $[F(1,8)=327.5, p<.001]$. The interaction between these two variables was not significant $[F(6,48)=$ $1.31, p=.257]$. A post hoc one-way ANOVA on each sound condition indicated that the bouncing percept increased as a function of PCT duration in the sound condition $[F(6,48)=3.79, p<.01]$ but did not in the no-sound condition $[F(6,48)=0.616, p=.716]$.

\section{Discussion}

Although the subjects were instructed to minimize the number of "unsure" responses, the difference in the certainty with and without a PCT was clear. When there was no trajectory after the visual target coincidence (i.e., PCT duration $=0 \mathrm{msec}$ ), the subjects were much less certain about their percept than when there was a nonzero PCT. Thus, a nonzero PCT is necessary for a clear perception of either streaming or bouncing motion event. Given that the task was to judge how the objects appeared to move after the moment of their coincidence, this sounds trivial. However, it confirmed that the subjects were able to notice the presence/absence of the PCT, that they were sure about their streaming/bouncing judgments in nonzero PCT conditions, and, therefore, that the percentage of bouncing reports is a reliable index of the subjects' perceptual bias in these cases. ${ }^{1}$

The main finding of Experiment 1 is that the bounceinducing effect of the sound did not fully develop at short PCT durations and reached the maximum level around the PCT of 150-200 msec. If the bounce-inducing effect of the sound was simply due to an explicit inference based on the physical relationship between the transient sound and the bouncing event, the presentation of the sound should have increased the frequency of the bouncing percept, independent of the PCT duration. Therefore, the results clearly reject the cognitive-bias hypothesis and suggest that the sound has to interact with visual motion mechanisms for some period after the coincidence in order to be maximally effective.

On the basis of the results of Experiment 1, we speculated that the interaction involved in the bounce-inducing 


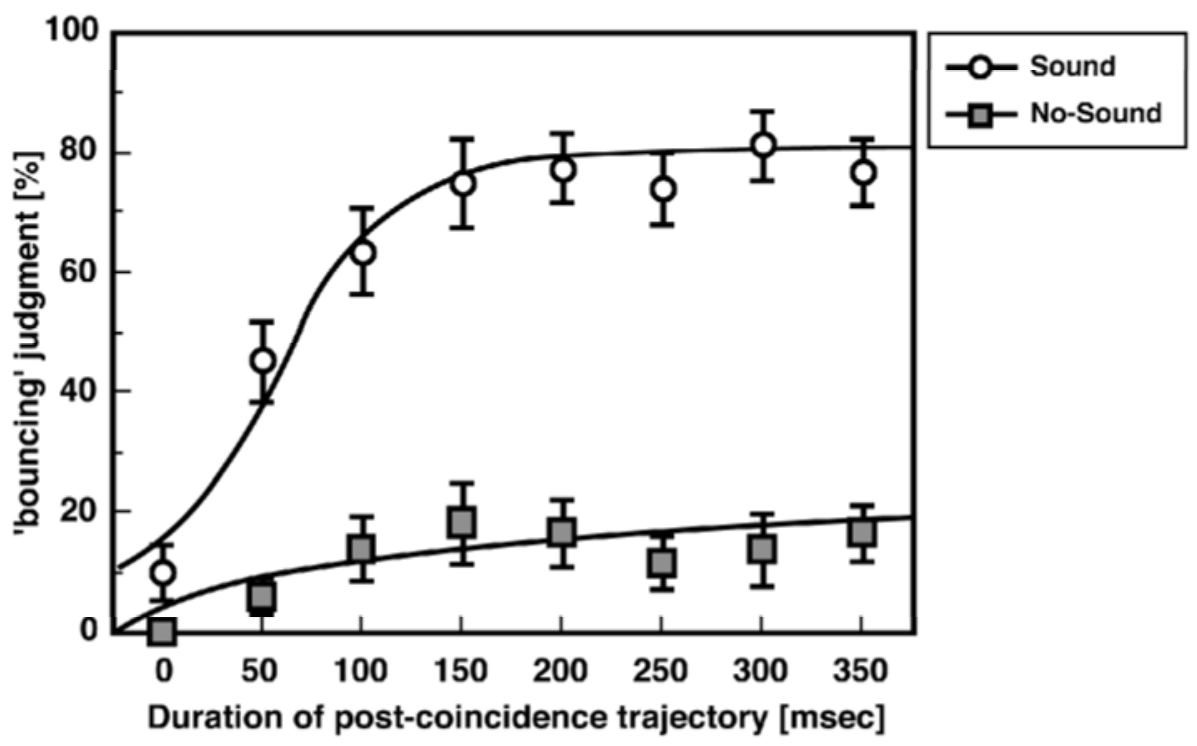

Figure 3. The effect of the PCT duration on the percentage of bouncing judgments in Experiment 1 . The vertical axis shows the averaged percentage of bouncing judgments $(N=9)$. The no-sound and sound conditions were fitted with a logarithmic function and a logistic function, respectively. T bars indicate $\pm 1 S E$.

effect of sound requires a PCT of about 150-200 msec to be completed. It is possible, however, that the distance of the PCT (i.e., how far the targets travel) is the important factor, rather than the duration of the PCT (i.e., how long the targets stay on). In Experiment 2, we aimed to dissociate contributions of the PCT duration and the PCT distance by doubling the speed of the moving targets.

\section{EXPERIMENT 2}

In Experiment 2, we employed the sound used in Experiment 1 as the bounce-inducing factor but doubled the speed (frame-to-frame displacement) of the visual targets. If a certain duration of PCT is required to fully derive the bouncing percept, the bounce-inducing effect should reach the maximum level at the same PCT duration as in Experiment 1 (about $150-200 \mathrm{msec}$ ). If the PCT distance was the primary factor, the PCT duration to maximize the bounce-inducing effect would become half of the duration observed in Experiment 1 (about 75-100 msec).

\section{Method}

Subjects. The same 9 subjects of Experiment 1 took part in Experiment 2.

Stimuli. The stimuli were almost identical to those used in Experiment 1 . However, the speed of the visual targets was doubled $\left(3.28^{\circ}\right.$ per second; frame-to-frame spatial offset $\left.=0.16^{\circ}\right)$, and the 350-msec PCT was not used.

Procedure. The procedure was almost the same as in Experiment 1 . There were seven different PCT durations $(0,50,100,150$, 200,250 , and $300 \mathrm{msec} ; 0^{\circ}, 0.16^{\circ}, 0.33^{\circ}, 0.49^{\circ}, 0.66^{\circ}, 0.82^{\circ}$, and $0.98^{\circ}$ in terms of PCT distance, respectively) and two sound condi- tions (no-sound or sound). Twenty trials were repeated for each combination of these conditions in random order (PCT duration or distance [7] $\times$ sound condition [2] $\times$ repeat [20] $=280$ trials). A full experiment was divided into four sessions, with 70 trials each.

\section{Results}

With the PCT equal to or over $50 \mathrm{msec}$, the percentages of "unsure" response were again quite low (about $8 \%$ for the 50-msec PCT and $1 \%$ for the other nonzero PCTs, averaged over subjects). Figure 4 portrays the averaged percentage of "bouncing" responses across all subjects as a function of PCT duration and the approximated curves. All the data in Experiment 2 were approximated well with logistic functions (no-sound condition, $R^{2}=.999$; sound condition, $R^{2}=.995$ ).

The following statistical analyses were conducted without the 0-msec PCT condition. A two-way ANOVA revealed main effects of PCT duration $[F(5,40)=7.28$, $p<.001]$ and sound condition $[F(1,8)=281, p<.001]$. The interaction did not show significance $[F(5,40)=1.07$, $p=.382]$. A one-way ANOVA on each sound condition showed that the percentage of the bouncing percept in the no-sound condition did not increase significantly as a function of PCT duration $[F(5,40)=1.82, p=.126]$, whereas it did increase in the sound condition $[F(5,40)=$ 5.81, $p<.001]$.

For comparison purposes, the results of Experiment 1 were also reapproximated with the range from 0 to $300 \mathrm{msec}$ and are shown in Figure 4. The results were fitted best with logistic functions (no-sound condition in Experiment $1, R^{2}=.774$; sound condition in Experi- 


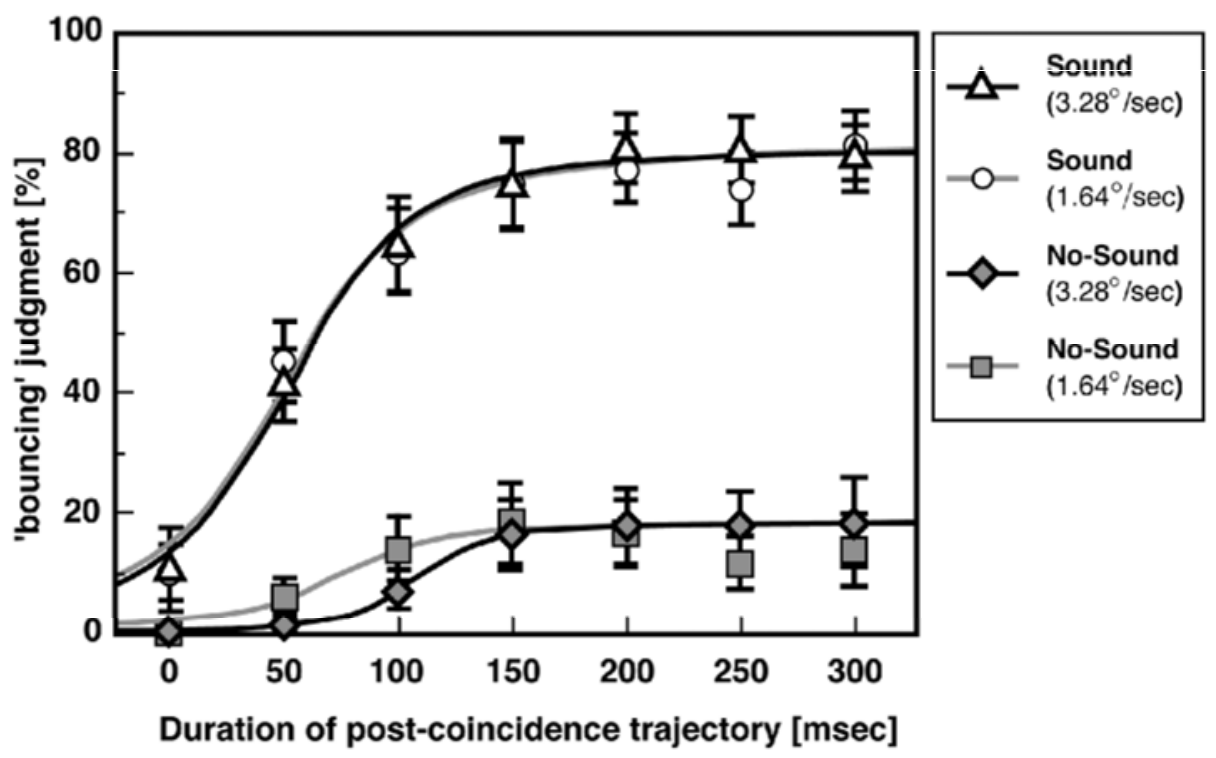

Figure 4. The effect of the PCT duration on the percentage of bouncing judgments in Experiment 2 (doubled speed). The vertical axis shows the averaged percentage of bouncing judgments $(N=9)$. The results of Experiment 2 were approximated with logistic functions. For comparison, the results of Experiment $1(0-300 \mathrm{msec})$ were reapproximated with logistic functions. $T$ bars indicate $\pm 1 S E$.

ment $\left.1, R^{2}=.979\right)$. The curves of the two sound conditions nearly overlie each other when they are plotted against PCT duration.

\section{Discussion}

Since the frame rate was fixed, the doubled speed of the moving disks in Experiment 2 resulted in a doubled PCT distance from the coincidence point at a given time, compared with those in Experiment 1. If the development of the bouncing percept depended on the distance of the PCT, the bouncing percept in Experiment 2 should have developed twice as fast as in Experiment 1, reaching the maximum level around 75-100 msec (leading to a leftward shift of the curve, compared with the curve of Experiment 1). However, the results show that the frequency of the bouncing percept increased as the same function of PCT duration in both Experiment 1 and Experiment 2. Therefore, we conclude that the PCT duration is indeed the determining factor for the development of the bouncing percept. The results of Experiment 2 strengthened the hypothesis that it takes about $150-200 \mathrm{msec}$ for the auditory stimulation to induce the bouncing percept maximally.

The PCT duration of about 150-200 msec may correspond to the delay for which information from the bounceinducing sound arrives at the level of auditory-visual interaction or the time for which auditory information fully interacts with visual information about the target motion from the PCT. In Experiment 3, we attempted to examine the first possibility that the slow development of the bouncing percept in our display is solely due to auditory transmission delay.

\section{EXPERIMENT 3}

R. Sekuler et al. (1997) reported that the bouncing percept was enhanced, though somewhat weakened, even when sound was presented a little $(250 \mathrm{msec}$ in their display) before or after the visual target coincidence. In Experiment 3, we took advantage of this tolerance for temporal asynchrony. Instead of presenting the exactly synchronized sound, we delivered the sound $100 \mathrm{msec}$ before or after the visual target coincidence and again varied PCT duration. If the delay itself in auditory process is the bottleneck that necessitates the PCT duration, the dependency of the sound-induced bouncing percept on the PCT duration would be different according to the shift of timing of sound presentation. To be more specific, the sound presented before the visual coincidence should reach its asymptote level faster than the sound presented at the moment of the visual coincidence.

\section{Method}

Subjects. The same 9 subjects in Experiments 1 and 2 took part in Experiment 3.

Stimuli. The visual stimuli were almost the same as those used in Experiment 1, except that we varied the PCT duration only from 50 to $300 \mathrm{msec}$. The single sound, identical to that used in Experiment 1 , was presented $100 \mathrm{msec}$ ( 2 frames) before or after the visual target superimposition.

Procedure. The procedure was the same as in Experiments 1 and 2. For each of the six PCT durations $(50,100,150,200,250$, and $300 \mathrm{msec}$ ) and each of two timings of sound presentation (soundbefore and sound-after), 20 trials were presented in random order (PCT duration [6] $\times$ sound timing [2] $\times$ repeat $[20]=240$ trials). A full experiment was composed of four sessions, with 60 trials each. 


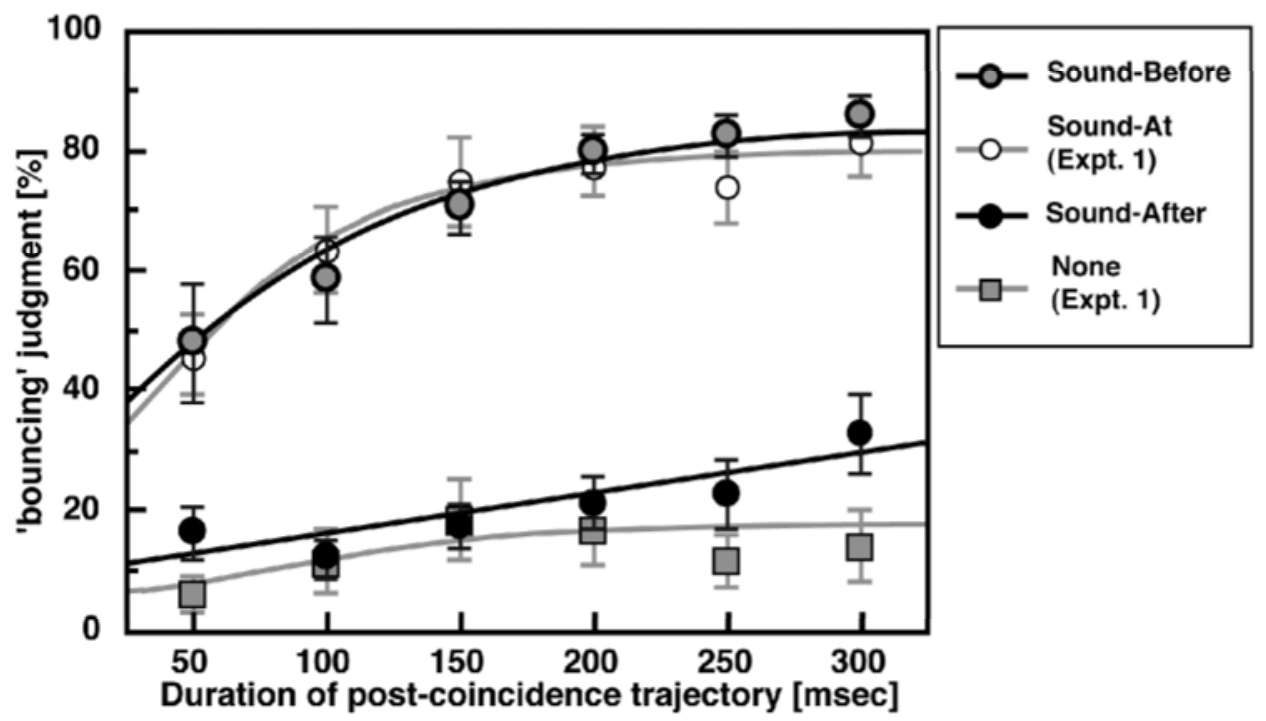

Figure 5. The effect of the PCT duration on the percentage of bouncing judgments in Experiment 3 (sound-before and sound-after). The vertical axis shows the averaged percentage of bouncing judgments $(N=9)$. The results of Experiment 3 and those of Experiment $1(50-300 \mathrm{msec})$ were plotted together for comparison. The sound-after condition was fitted with a linear function, and the other conditions were fitted with logistic functions. $T$ bars indicate $\pm 1 S E$.

\section{Results}

The subjects produced few (about $4 \%$ in the entire trials) "unsure" responses throughout the experiment. Figure 5 shows the results of Experiment 3 (sound-before and sound-after), together with the results of Experiment 1 (no-sound and sound-at). The results of Experiment 3 and those of Experiment 1 (PCT range from 50 to $300 \mathrm{msec}$ ) were curve-fitted in the same way as in Experiment 1. The sound-after condition of Experiment 3 was fitted with a linear function $\left(R^{2}=.777\right)$, and the data of the other conditions were approximated with logistic functions (nosound condition of Experiment $1, R^{2}=.471$; sound-before condition of Experiment 3, $R^{2}=.965$; sound-at condition of Experiment 3, $R^{2}=.968$ ). For the results of Experiment 3, a two-way ANOVA was conducted. Significant main effects of PCT duration $[F(5,40)=7.64, p<.001]$ and sound timing [sound-before vs. sound-after, $F(1,8)=$ $269.4, p<.001]$ were found. The interaction between these factors was not significant $[F(5,40)=1.89, p=.104]$.

The sound presented $100 \mathrm{msec}$ before the visual target coincidence increased the frequency of the bouncing percept. The bounce-inducing effect of the preceding sound grew as PCT duration increased [one-way ANOVA, $F(5,40)=6.48, p<.001]$ in a time course quite similar to that of the sound-at condition in Experiment 1.

When the sound delayed the visual target coincidence by $100 \mathrm{msec}$, the bouncing percept seemed to be enhanced, but only slightly, compared with the no-sound condition of Experiment 1. With the delayed sound, however, the effect of PCT duration did not reach a significant level [oneway ANOVA, $F(5,40)=2.18, p=.072]$ in Experiment 3 .

\section{Discussion}

Consistent with R. Sekuler et al. (1997), even when the sound lagged the visual target coincidence by $100 \mathrm{msec}$, the sound presentation seems to have weakly promoted the bouncing percept at the longer PCTs. Thus, motion perception may be affected by auditory stimulation that occurs within a broad-tuned interaction range, although sound before the visual event has a greater effect and a broader range of interaction than sound after the visual event (R. Sekuler et al., 1997; Watanabe, Scheier, Lewkowicz, \& Shimojo, 1999; Watanabe, Scheier, \& Shimojo, 1999).

The sound presented $100 \mathrm{msec}$ before the visual target coincidence produced almost the same effect and dependency on the PCT duration as the sound presented at the visual target coincidence. This result suggests that the delay of the bounce-inducing effect of sound is not simply due to a delay in the auditory processing. Therefore, we conclude that some interactive processes between visual information from the PCT and auditory information may be required for the bounce-inducing effect of sound, which would take approximately 150-200 msec independently of the visual target speed.

We take the PCT dependency of the induced bouncing percept as evidence against the cognitive-biashypothesis, since the explicit mapping from the sound presentation to the bouncing percept would result in no PCT dependency. Additionally, we observed that $150-200 \mathrm{msec}$ is required for interactions between the bounce-inducing sound and the PCT to reach the steady state of the bouncing percept. However, these observations may be specific to the bounce- 


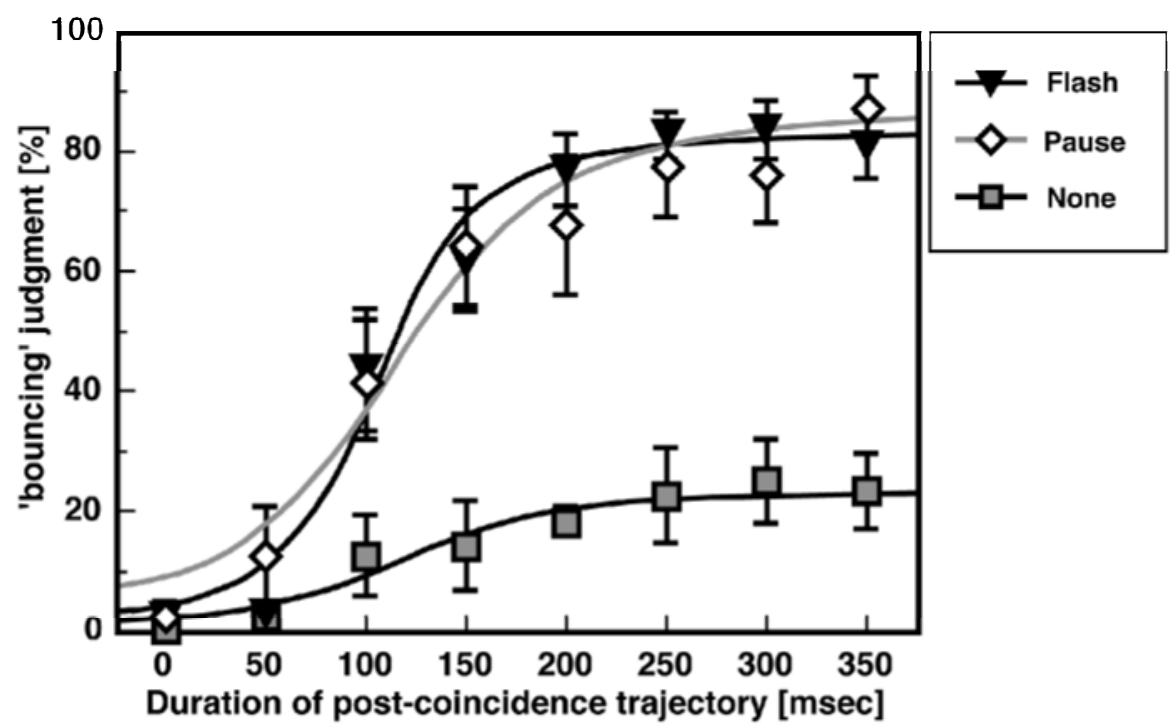

Figure 6. The effect of the PCT duration on the percentage of bouncing judgments in Experiment 4 . The vertical axis shows the averaged percentage of bouncing judgments $(N=6)$. The none condition was approximated with a logarithmic function, and the flash and pause conditions were fitted with logistic functions. $T$ bars indicate $\pm 1 S E$.

inducing effect of sound. In Experiments 4 and 5, we attempted to examine whether the same results would be obtained by using other bounce-inducing factors that are not auditory.

\section{EXPERIMENT 4}

Transient visual factors, such as a momentary pause of the moving targets (Bertenthal et al., 1993) and an abrupt visual distractor (Watanabe \& Shimojo, 1998) at the time of the target coincidence, have also been reported to produce the bounce-inducing effect. In Experiment 4, we examined (1) whether the bounce-inducing effect with these visual factors shows the PCT dependency and, if so, (2) whether the PCT dependency is similar to that observed with the sound in the previous experiments.

\section{Method}

Subjects. Six of the subjects from Experiment 1 (including the authors) participated.

Stimuli. Since the experiment was conducted at a different site and with a different experimental setup, a few stimulus parameters were slightly different from Experiment 1 . Stimuli were presented by a Silicon Graphics Iris workstation. The luminance of the gray background was $10.8 \mathrm{~cd} / \mathrm{m}^{2}$. The observation distance was $60 \mathrm{~cm}$. The other stimulus parameters, such as the stimulus size and the speed of the visual targets, were kept identical to those of Experiment 1 . In one third of the trials, the visual targets stopped at the location of the coincidence for two frames (100 msec; pause). In another third of the trials, when the visual targets were superimposed, a black ring $\left(0.01 \mathrm{~cd} / \mathrm{m}^{2}, 0.8^{\circ}\right.$ in diameter $)$ was flashed for one frame around the location of the target coincidence (flash). In the rest of the trials, no specific event occurred at the visual target coincidence (none).

Procedure. The three-alternativ e forced-choice paradigm (streaming, bouncing, or unsure) was used. For each of the eight PCT du- rations $(0,50,100,150,200,250,300$, and $350 \mathrm{msec})$ and three conditions of visual factor (none, pause, flash), 20 trials were presented in random order (PCT duration [8] $\times$ visual factor condition [3] $\times$ repeat [20] $=480$ trials). A full experiment was composed of four sessions, with 120 trials each.

\section{Results}

As in the previous experiments, the percentage of "unsure" responses was negligible (about $2 \%$ ) when there was a nonzero PCT. The averaged percentage of bouncing reports in Experiment 4 is presented in Figure 6. Logistic functions were used for approximation because they fitted the data best (none, $R^{2}=.955$; pause, $R^{2}=.967$; flash, $\left.R^{2}=.980\right)$.

Again, the following statistical analyses were all conducted without the 0 -msec PCT condition. With the none and pause conditions, we found main effects of both PCT duration $[F(6,30)=9.458, p<.001]$ and visual factor [none vs. pause, $F(1,5)=115.4, p<.001]$. The interaction was also significant $[F(6,30)=2.85, p<.05]$. Similarly, with the none and flash conditions, main effects of PCT duration $[F(6,30)=18.16, p<.001]$ and visual factor [none vs. flash, $F(1,5)=180, p<.001$ ] and a significant interaction $[F(6,30)=2.85, p<.05]$ were found. Between the pause and flash conditions, we found no difference [PCT, $F(6,30)=25.11, p<.001$; pause vs. flash, $F(1,5)=0.020, p=.887$; interaction, $F(6,30)=$ $0.434, p=.854]$.

Post hoc analyses with a one-way ANOVA on each condition of visual factor showed that the bouncing percept increased as a function of PCT duration in the pause condition $[F(6,30)=8.19, p<.001]$ and in the flash condition $[F(6,30)=21.88, p<.001]$ but did not in the none condition $[F(6,30)=1.69, p=.152]$. 


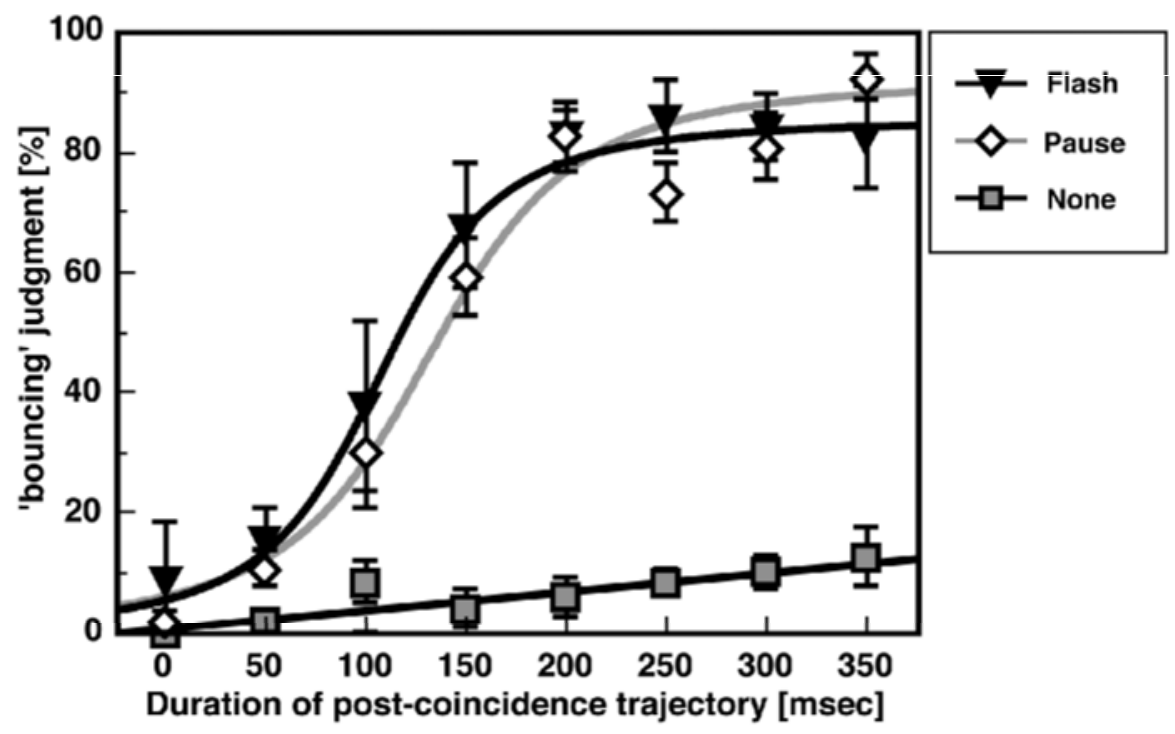

Figure 7. The effect of the PCT duration on the percentage of bouncing judgments in Experiment 5 (doubled speed). The vertical axis shows the averaged percentage of bouncing judgments $(N=6)$. The none condition was approximated with a linear function, and the flash and pause conditions were fitted with logistic functions. $T$ bars indicate $\pm 1 S E$.

\section{Discussion}

Both the abrupt visual distractor and the $100-\mathrm{msec}$ pause of the visual targets at the coincidence point significantly increased the frequency of the bouncing percept, if the PCT duration was long enough. In Experiment 4, the development of the bouncing percept, induced by both of the visual bounce-inducing factors, depended on the duration of PCT up to around 150-200 msec, whereas the number of "unsure" responses are quite low, similar to that in Experiment 1. Therefore, it seems that the bounceinducing effects of pause and of flash were not due to a cognitive bias, but they require interactions between the bounce-inducing factors and the PCT as the bounceinducing effect of sound (Experiment 1). In Experiment 5, we sought to further confirm that the visually induced bouncing percept depended not on the PCT distance but on the PCT duration.

\section{EXPERIMENT 5}

We employed the same visual bounce-inducing factors (pause and flash) as in Experiment 4, but we doubled the visual target speed, as we did in Experiment 2. Logic of the experiment was the same as that of Experiment 2. If the PCT distance would determine the development of the bounce-inducing effect, results should have exhibited a leftward shift of the curves in Figure 6.

\section{Method}

Subjects. The same 6 subjects as in Experiment 3 participated.

Stimuli. The stimuli were identical to those used in Experiment 4 , except that the speed of the visual targets was doubled $\left(3.28^{\circ}\right.$ per second; frame-to-frame spatial offset $\left.=0.16^{\circ}\right)$.

Procedure. The procedure was the same as in Experiment 4.

\section{Results}

With nonzero PCT durations, the number of "unsure" responses was small (about $4 \%$ of the entire trials), and, therefore, it can be disregarded except for the 0 -msec PCT condition. Figure 7 gives the results of Experiment 4 in the form of the averaged percentage of bouncing responses as a function of PCT duration. The results of the none condition were fitted with a linear function $\left(R^{2}=\right.$ $.807)$, and the results of the pause and flash conditions were fitted with logistic functions (pause, $R^{2}=.970$; flash, $R^{2}=.939$ ).

Two-way ANOVAs, without the 0-msec PCT condition, resulted in statistical conclusions almost identical to those in Experiment 3 . For the none and pause conditions, main effects of both PCT duration $[F(6,30)=21.68$, $p<.001$ ] and visual factor [none vs. pause, $F(1,5)=$ $326.6, p<.001]$ were found. The interaction was also significant $[F(6,30)=17.02, p<.001]$. A similar pattern was obtained with the none and flash conditions [PCT, $F(6,30)=12.51, p<.001 ;$ none vs. flash, $F(1,5)=241.9$, $p<.001$; interaction, $F(6,30)=9.68, p<.001]$. There was no difference between the pause and flash conditions [PCT, $F(6,30)=31.84, p<.001$; pause vs. flash, $F(1,5)=$ $2.089, p=.154$; interaction, $F(6,30)=0.175, p=.971]$.

The bounce-inducing effect of both visual factors increased as a function of PCT duration [one-way ANOVA; pause, $F(6,30)=23.06, p<.001$; flash, $F(6,30)=12.19$, $p<.001]$. When there were no visual bounce-inducing factors, the streaming percept dominated over all PCT durations $[F(6,30)=1.246, p=.313]$.

\section{Discussion}

We did not superimpose the results of Experiment 4 on the results of Experiment 5 in order to avoid a crowd- 
ing presentation. However, it is clear that the results of Experiment 5 were almost the same as those of Experiment 4, despite the doubled speed of the visual targets. Since this similarity appears when the results are plotted as functions of PCT duration, the PCT duration, not the PCT distance, is the primary factor for the development of the bouncing percept induced by visual factors. Thus, we observed similar results between the auditory bounceinducing factors and the visual bounce-inducing factors. Both auditory and visual factors depend on the PCT duration and require a PCT of about $150-200 \mathrm{msec}$ to induce the bouncing percept.

Why does the bouncing percept take time to develop in our display? One possible explanation is that it is because our display may intrinsically favor the streaming percept. Bouncing events in the natural environment have spatiotemporal characteristics different from streaming events. Objects reverse their motion direction as soon as their outer contours contact each other. Our streaming/bouncing motion display included frames against this physical constraint (the overlapping frame and one of the touching frames; hereafter called the coincidence frames) and, thus, the timing of motion reversal should be later than the physical bouncing. If the coincidence frames are removed from our display, then the display would more closely resemble physical bouncing events of solid objects. Many studies showed that such a display easily led to a exclusive bouncing percept (e.g., Flynn, 1994; Gilden \& Proffitt, 1989, 1994; Kaiser \& Proffitt, 1987; Runeson \& Vedeler, 1993; Todd \& Warren, 1982), though in a different context of research concerning the kinematic information acquisition of simulated physical collision events. Therefore, if the coincidence frames were the main cause of the streaming dominance and of the slow development of bouncing percept, the removal of those frames might produce results totally different from the results of the present experiments (i.e., a high overall percentage of the bouncing percept). In Experiment 6, we examined this possibility.

\section{EXPERIMENT 6}

\section{Method}

Subjects. The same 6 subjects as in Experiment 3 participated.

Stimuli. The stimuli were identical to those used in Experiment 4 , except the overlapping frame and one of the touching frames (coincidence frames) were removed so that the visual display would more closely mimic bouncing events. The flash and pause were presented when the two disks touched each other.

Procedure. The procedure was the same as in Experiment 4.

\section{Results}

The percentage of "unsure" responses was quite low when there was a nonzero PCT (about $3 \%$ of the entire trials). Figure 8 shows the averaged percentage of "bouncing" responses across all subjects as a function of PCT duration and the approximated curves. The none condition was approximated best with a logarithmic function $\left(R^{2}=\right.$ $.865)$, and the other conditions were fitted best with a logistic function (pause, $R^{2}=.965$; flash, $R^{2}=.957$ ).
The following statistical analyses were all conducted without the 0 -msec PCT condition. When the none and pause conditions were compared, we found significant main effects in both visual factors [none vs. pause, $F(1,5)=$ $115.4, p<.01]$ and PCT duration $[F(6,30)=9.45, p<$ $.01]$. The interaction showed significance $[F(6,30)=2.85$, $p<.05]$. Likewise, with the none and flash conditions, main effects of PCT duration $[F(6,30)=25.16, p<.01]$ and the visual factor $[F(1,5)=22.04, p<.01]$ and a significant interaction $[F(6,30)=1.79, p<.01]$ were found. Between the pause and flash conditions, we also found a significant difference [pause vs. flash, $F(1,5)=0.02, p<$ $.01]$, but the interaction was not significant $[F(6,30)=$ $0.434, p=.1]$. Post hoc analyses with a one-way ANOVA on each condition of visual factor showed that the bouncing percept increased as a function of PCT duration in the pause condition $[F(6,30)=4.44, p<.01]$ and in the flash condition $[F(6,30)=6.59, p<.01]$ but did not in the none condition $[F(6,30)=0.891, p=.512]$.

\section{Discussion}

Compared with the results of Experiment 4, the removal of the coincidence frames in Experiment 6 had no effect when there was no bounce-inducing factor and when the flash induced the bouncing percept. When the pause was introduced at the moment when the two disks touched, the bouncing percept was enhanced substantially. But this may be simply because the subjects were given more time to notice that the two disks did not overlap each other when the pause was present. That is, the interaction between the pause and the removal of the coincidence frames might introduce another factor that promotes the bouncing percept: the appreciable perception of "nonoverlapping." This clear perception of nonoverlapping of the visual target may be a strong visual cue signaling bouncing events.

The mimicking of the bouncing event failed to promote the bouncing percept in our display probably because the size of the visual stimuli $\left(0.16^{\circ}\right)$ was much smaller than those used in conventional collision studies (e.g., $1.0^{\circ}$ in Kaiser \& Proffitt, 1987). This was confirmed in our additional observation by using a larger moving target $\left(1.28^{\circ}\right)$ without the coincidence frames. If the size of the disks was large enough, as in the conventional collision studies, the subjects always interpreted the visual display as the bouncing event even without a bounce-inducing factor, presumably due to the noticeable elimination of the coincidence frames mentioned above. With the large size of the disk, when the coincidence frames were present, the subjects almost always perceived the streaming event. The dependency on the size of moving objects in the streaming/ bouncing event perception is interesting because it suggests that there is a size threshold for figural event information to affect motion event perception. Nonetheless, the size dependency does not urge us to change our interpretation of the results of Experiments 1-5.

The results of Experiment 6 suggest that the PCT dependency of the bouncing percept was not solely due to the intrinsic visual cues in our display. Additionally, if the 


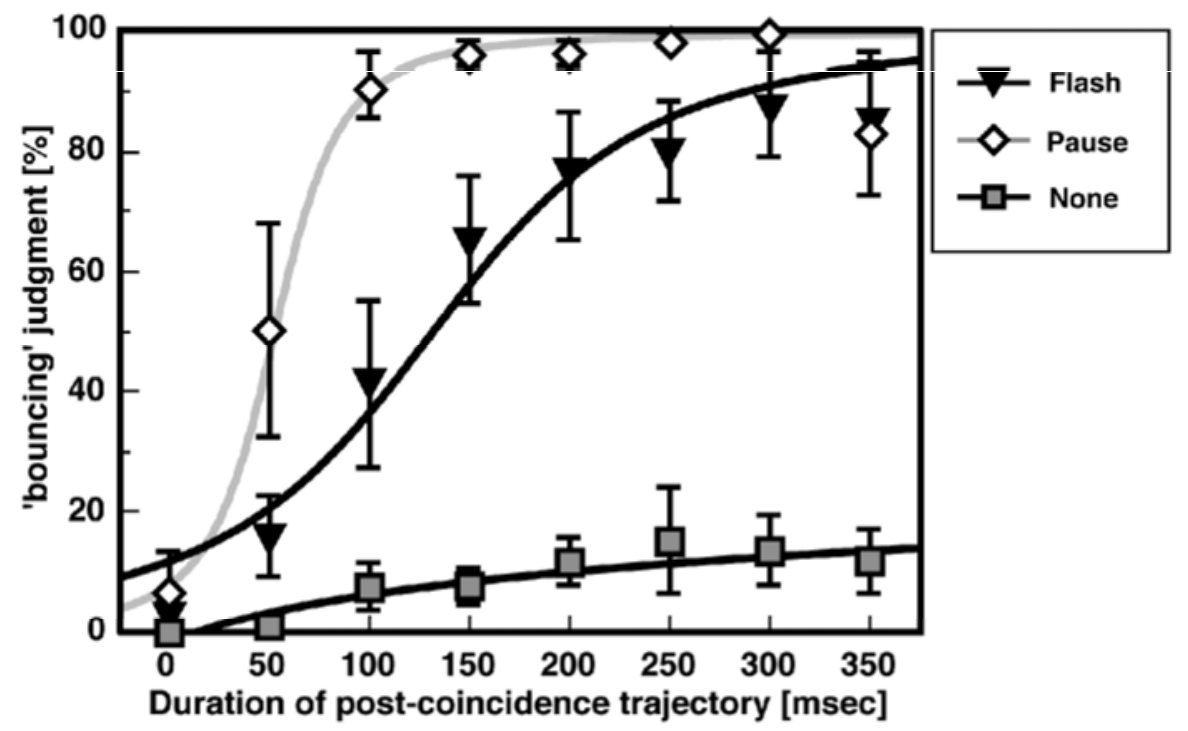

Figure 8. The effect of the PCT duration on the percentage of bouncing judgments in Experiment 6 (coincidence frame removal). The vertical axis shows the averaged percentage of bouncing judgments $(N=6)$. The none condition was approximated with a logarithmic function, and the flash and pause conditions were fitted with logistic functions. T bars indicate $\pm 1 S E$.

presence of the coincidence frames enhanced the streaming percept in our original display and prevented the bouncing percept from developing quickly, the enhancement of the bouncing percept with the 100-msec pause in Experiments 4 and 5 would be puzzling. This is because the subject must notice the coincidence frames (which allegedly serve as strong cues for streaming) more frequently with the pause than without it. Therefore, we argue that the coincidence frames were not the main cause of the dominance of the streaming percept in our display.

\section{GENERAL DISCUSSION}

In the present study, we investigated how the bounceinducing effect depends on the duration of the trajectory after the visual coincidence. The main finding is that the bouncing percept was not consolidated immediately after the coincidence, but it develops as a function of PCT duration up to $150-200 \mathrm{msec}$. This does not mean that the subjects simply became more certain about their percept, since the number of "unsure" responses was negligible when there was a minimal PCT duration $(\geq 50 \mathrm{msec})$ in all the experiments. In other words, at short PCTs, the percept was clear, but it was not the bouncing percept (i.e., the streaming percept). We interpret the results as rejecting that the bounce-inducing effect is merely a cognitive bias.

Apparently, all bounce-inducing effects follow the similar PCT-duration dependency of the bouncing percept, irrespective of the modality of the bounce-inducing factor. Additionally, for the bounce-inducing effect of sound, the earlier registration of the bounce-inducing fac- tor did not affect the PCT-duration dependency (Experiments 1 and 3), suggesting that the interaction between the bounce-inducing sound and the PCT is critical. Also, between the pause and the flash (visual factors), the development of the bouncing effect is similar (Experiments 4 and 5). These results converge into the hypothesis that some (intramodal or intermodal) interactions between information from the PCT and information from the bounceinducing factor are required for the brain to interpret the streaming/bouncing display as a bouncing event. Such interactions may take time up to $150-200 \mathrm{msec}$, as reflected in the PCT-duration dependency of the bouncing percept.

\section{Interactions Between Bounce-Inducing \\ Factors and Visual Processes for \\ Directional Information Extraction}

The results of Experiment 6 suggest that the coincidence frames in our display may not be the main cause of the streaming dominance and that of the slow development of the bouncing percept. Another possible origin of the PCT dependency of the bouncing percept would be interactions between the bounce-inducing factors and the process with which the visual system determines change or unchange in speed and/or direction of visual motion. It has been reported that directional information extraction is a time-consuming task for the visual motion system (Bowne, McKee, \& Glaser, 1989; A. B. Sekuler, R. Sekuler, \& E. B. Sekuler, 1990; Watamaniuk, R. Sekuler, \& Williams, 1989). The delay for determining target velocity is probably a result of velocity signals being smoothed over time due to temporal or sequential recruitment (Bowne et al., 1989; Casco \& Morgan, 1987; Lap- 
pin \& Bell, 1976; McKee \& Welch, 1985; Nakayama \& Silverman, 1984; Snowden \& Braddick, 1991).

Two kinds of temporal "recruitment" of motion were known: one for the integration of motion signals within a single detector and the other for that across detectors. Psychophysical and modeling studies have estimated the temporal integration time for motion direction interaction, but the estimated integration time varies among studies, mainly because the tasks involved (and perhaps the underlying mechanisms) were different. For simple discrimination of motion direction, the temporal integration time was estimated to be less than $150 \mathrm{msec}$ (e.g., Fredericksen, Verstraten, \& van de Grind, 1994; Watson, 1979; Watson \& Turano, 1995). Also, physiologically, temporal integration times for directional interactions in V1 and MT cells of macaques are on the order of $100 \mathrm{msec}$ (Mikami, Newsome, \& Wurtz, 1986). These values may correspond to temporal recruitment within a single motion detector. On the other hand, by using noisy motion conditions or by introducing directional uncertainty of motion, other studies have reported longer integration times ranging from about 200-700 msec (e.g., Bowne et al., 1989; A. B. Sekuler \& R. Sekuler, 1993; A. B. Sekuler et al., 1990; Snowden \& Braddick, 1991; Watamaniuk, McKee, \& Grzywacz, 1995; Watamaniuk \& R. Sekuler, 1992; Watamaniuk et al., 1989), perhaps corresponding to the period with which the process of temporal recruitment across multiple motion detectors attains a stable solution under noises.

The streaming/bouncing motion configuration may be considered to be a variation of a noisy motion condition because there is directional ambiguity. Our estimate of the time necessary for the maximal bounce-inducing effect (150-200 msec) falls between the longest integration times for a single local-motion detector and the shortest integration time among multiple local-motion detectors. The detection of the bouncing percept may be thought of as the easiest case of directional information extraction through the use of multiple local-motion detectors. It is easy probably because only a binary decision is required and because the perceptual state is biased toward "direction change" (bouncing) by the bounce-inducing factors before and/or during directional information extraction processes.

On the basis of the similar time course, we infer that the common mechanisms for directional information extraction may underlie the PCT-duration dependency of the bouncing percept. The hypothesis can explain why various bounce-inducing factors take approximately the same amount of the PCT duration to maximize their effects. With directional ambiguity, the interaction between the bounce-inducing factors and visual process(es) dealing with the PCT is required to finally construct the bouncing percept.

Bertenthal et al. (1993) reported that disparity information had effects on the relative frequency of each percept. A. B. Sekuler and R. Sekuler (1999) have shown that occlusion at the visual coincidence did not alter the streaming/bouncing motion perception and suggested that an explanation based solely on the low-level processing of visual motion is unsatisfactory. Finally, Watanabe and Shimojo (1998) demonstrated that the relative frequency of the streaming/bouncing motion perception can be modulated by the observer's attentional state.

Given the results of these studies, the processing level of the streaming/bouncing percept should be at least higher than the level of the first-order (luminance-defined or Fourier) motion (Lu \& Sperling, 1995), since the firstorder motion mechanism has been reported to be primarily monocular and presumably operates without an attentional process. Possible mechanisms for the streaming/bouncing motion event perception-namely, mechanisms for directional information extraction-can be at the level of attentional motion tracking (Cavanagh, 1992).

\section{Perception as a Hypothesis-Testing Process}

Finally, we think it is likely that the streaming percept dominates in our display because it represents the majority of physical events (Ramachandran \& Anstis, 1983), given the retinal input of the streaming/bouncing motion display.

A coincidence of two retinal moving images rarely implies a coincidence of two objects in three-dimensional space. This is because (1) mathematically, there are infinite ways to project a point in a three-dimensional space onto a two-dimensional surface such as the retina, (2) physically, objects are seldom aligned at the same depth plane, and (3) due to momentum, objects do not usually change speed or direction abruptly. Thus, in the natural environment, the conditional probability of streaming events given this two-dimensional retinal projection of visual stimuli would be much higher than that of bouncing events. The perceptual system may compute these probabilities and behave as if these events occur accordingly (the generic view principle and Bayesian modeling; e.g., Freeman, 1994; Knill, Kersten, \& Yuille, 1996; Nakayama \& Shimojo, 1992; Richards, 1988; Richards, Koenderink, \& Hoffman, 1987). In other words, the streaming percept is generic. In contrast, the bouncing event is accidental: It would happen only when the two moving objects exist in the same depth plane. This interpretation is consistent with the finding that the bouncing percept is more frequently perceived with binocular observation than with monocular observation (Sumi, 1995). Binocular observation provides disparity information that the objects exist in the same depth plane, whereas monocular observation provides no such information.

Moreover, in a natural environment, changes in direction and speed of the objects (postcollision scatter) usually specify the occurrence of a physical collision. In order for a physical collision to produce the kinematics of the two-dimensional display used in the present study, the two objects must approach each other completely in line and the collision must be perfectly central, which is highly unlikely-hence, accidental.

Thus, the perceptual system may tend to interpret the visual display as the streaming event for these reasons, unless there is a suggestive clue, such as a transient and 
synchronized auditory (sound) or visual (pause or flash) event. Although how these events alter motion perception remains unsolved and needs to be investigated further, the present study demonstrated that there are similar time courses for the bounce-inducing cues to be maximally effective and thus suggested that there may be a common process for the streaming/bouncing motion event perception.

\section{Conclusion}

The present study demonstrated that the simple cognitive-bias explanation cannot account for the bounceinducing effects of sound, pause, and flash and that the brain may utilize multiple cues from different sources to derive the apparently same category of events-namely, "bouncing." Some period of interactions between the bounce-inducing factors and visual information from the PCT seems to be necessary to derive the bouncing percept, which is estimated to be approximately $150-200 \mathrm{msec}$ in the present experiments. In order to clarify what processes occur during this temporal range and how the bounceinducing factors interact with the PCT, more investigations are warranted.

\section{REFERENCES}

American Psychological Association. (1992). Ethical principles of psychologists and code of conduct. American Psychologist, 47, 1597 1611 .

Bertenthal, B. I., Banton, T., \& Bradbury, A. (1993). Directional bias in the perception of translating patterns. Perception, 22, 193-207.

Bertenthal, B. I., \& Kramer, S. J. (1988). Perceptual constraints on the direction of moving surfaces [Abstract]. Investigative Ophthalmology \& Visual Science, 29, S250.

Bowne, S. F., McKee, S. P., \& Glaser, D. A. (1989). Motion interference in speed discrimination. Journal of the Optical Society of America, 64, 1715-1719.

Casco, C., \& Morgan, M. J. (1987). Detection of moving local density differences in dynamic random patterns by human observers. Perception, 16, 711-712.

CAVANAGH, P. (1992). Attention-based motion perception. Science, 257, 1563-1565.

Cavanagh, P., Arguin, M., \& von Grunau, M. (1989). Interattribute apparent motion. Vision Research, 29, 1197-1204.

FLYNN, S. B. (1994). The perception of relative mass in physical collisions. Ecological Psychology, 6, 185-204.

Fredericksen, R. E., Verstraten, F. A. J., \& van de Grind, W. A. (1994). Spatial summation and its interaction with the temporal integration mechanism in human motion perception. Vision Research, 34, 3171-3188.

Freeman, W. T. (1994). The generic viewpoint assumption in a framework for visual perception. Nature, 368, 542-545.

Gilden, D. L., \& Proffitt, D. R. (1989). Understanding collision dynamics. Journal of Experimental Psychology: Human Perception \& Performance, 15, 372-383.

Gilden, D. L., \& Proffitt, D. R. (1994). Heuristic judgment of mass ratio in two-body collisions. Perception \& Psychophysics, 56, 708720.

Goldberg, D. M., \& Pomerantz, J. R. (1982). Models of illusory pausing and sticking. Journal of Experimental Psychology: Human Perception \& Performance, 8, 547-561.

Gorea, A., \& Labarre, J. (1997a). A simplified, low level account of the bistable perception yielded by objects drifting toward and past one another [Abstract]. Perception, 26(Suppl.), 90.

Gorea, A., \& Labarre, J. (1997b). Streaming and bouncing: A low level account [Abstract]. Investigative Ophthalmology \& Visual Science, 38, S693.
Johansson, G. (1950). Configurations in the perception of velocity. Acta Psychologica, 7, 25-79.

Kaiser, M. K., \& Proffitt, D. R. (1987). Observers' sensitivity to dynamic anomalies in collisions. Perception \& Psychophysics, 42, 275 280.

Knill, D. C., Kersten, D., \& Yuille, A. (1996). Introduction: A Bayesian formulation of visual perception. In D. C. Knill \& W. Richards (Eds.), Perception as Bayesian inference (pp. 1-21). Cambridge: Cambridge University Press.

LAPPIN, J. S., \& BELL, H. H. (1976). The detection of coherence in moving random-dot patterns. Vision Research, 16, 161-168.

Lu, Z.-L., \& SPERLING, G. (1995). The functional architecture of human visual-motion perception. Vision Research, 35, 2697-2722.

McKeE, S. P., \& Welch, L. (1985). Sequential recruitment in the discrimination of velocity. Journal of the Optical Society of America A, 2, 243-251.

Metzger, W. (1934). Beobachtungen über phänomenale Identiät [Observations regarding phenomenal identity]. Psychologische Forschung, 19, 1-60.

Michotте, A. (1963). The perception of causality (T. R. Miles \& E. Miles, Trans.). Andover, U.K.: Methuen. (Original work published 1946)

Mikami, A., Newsome, W. T., \& Wurtz, R. H. (1986). Motion selectivity in macaque visual cortex: II. Spatiotemporal range of directional interactions in MT and V1. Journal of Neurophysiology, 55, 1328-1339.

Nakayama, K., \& Shimojo, S. (1992). Experiencing and perceiving visual surfaces. Science, 257, 1357-1363.

Nakayama, K., \& Silverman, G. H. (1984). Temporal and spatial characteristics of the upper displacement limit for motion in random dots. Vision Research, 24, 293-299.

Ramachandran, V. S., \& Anstis, S. M. (1983). Extrapolation of motion path in human visual perception. Vision Research, 23, 83-85.

Richards, W. (1988). The approach. In W. Richards (Ed.), Natural computation (pp. 3-17). Cambridge, MA: MIT Press.

Richards, W., KoEnderinK, J. J., \& HofFMan, D. D. (1987). Inferring three-dimensional shapes from two-dimensional silhouettes. Journal of the Optical Society of America A, 4, 1168-1175.

Runeson, S., \& Vedeler, D. (1993). The indispensability of precollision kinematics in the visual perception of relative mass. Perception \& Psychophysics, 53, 617-632.

Sekuler, A. B., \& Sekuler, R. (1993). Representational development of direction in motion perception: A fragile process. Perception, 22, 899-915.

SeKuler, A. B., \& Sekuler, R. (1999). Collisions between moving visual targets: What controls alternative ways of seeing an ambiguous display? Perception, 28, 415-432.

Sekuler, A. B., Sekuler, R., \& Sekuler, E. B. (1990). How the visual system detects changes in the direction of moving targets. Perception, 19, 181-195.

Sekuler, R, Sekuler, A. B., \& Brackett, T. (1995). When visual objects collide: Repulsion and streaming [Abstract]. Investigative Ophthalmology \& Visual Science, 36, S50.

Sekuler, R., Sekuler, A. B., \& Lau, R. (1997). Sound alters visual motion perception. Nature, $\mathbf{3 8 5}, 308$.

Snowden, R. J., \& BRAdDick, O. J. (1991). The temporal integration and resolution of velocity signals. Vision Research, 31, 907-914.

Sumi, S. (1995). Bounce effect seen in opposite motions. Japanese Psychological Research, 37, 195-200.

TodD, J. T., \& WARREN, W. H. (1982). Visual perception of relative mass in dynamic events. Perception, 11, 325-335.

Ullman, S. (1979). The interpretation of visual motion. Cambridge, MA: MIT Press.

Watamaniuk, S. N. J., McKee, S. P., \& Grzywacz, N. M. (1995). Detecting a trajectory embedded in random-direction motion noise. $\mathrm{Vi}$ sion Research, 35, 65-77.

Watamaniuk, S. N. J., \& SeKuler, R. (1992). Temporal and spatial integration in dynamic random dot stimuli. Vision Research, 32, 931-941.

Watamaniuk, S. N. J., Sekuler, R, \& Williams, D. W. (1989). Direction perception in complex dynamic displays: The integration of direction information. Vision Research, 29, 47-59.

Watanabe, K., Scheier, C., Lewkowicz, D. J., \& Shimojo, S. (1999, 
October). Temporal window for audio-visual interaction revealed with an ambiguous motion display. Poster presented at the Experimental Psychology Society Workshop on Crossmodal Attention and Multisensory Integration, Oxford.

Watanabe, K., Scheier, C., \& Shimojo, S. (1999, March). Temporal window for audio-visual integration revealed with bistable motion display. Paper presented at the Joint Symposium on Frontiers in Computational Neuroscience, Elat, Israel.

Watanabe, K., \& Shimojo, S. (1998). Attentional modulation in perception of visual motion events. Perception, 27, 1041-1054.

Watson, A. B. (1979). Probability summation over time. Vision Research, 19, 515-522.

Watson, A. B., \& Turano, K. (1995). The optimal motion stimulus. Vision Research, 35, 325-336.

\section{NOTE}

1. Two control experiments were conducted to examine the possible effect of demand characteristics of the task. In the first control experiment, a different set of subjects $(N=9)$ ran the same experiments with- out the demand of minimization of "unsure" responses. We found that this difference in instructions did increase the number of unsure responses at the 0-msec PCT but did not make any difference with longer PCTs. In the second control experiment, in a single session, only 350msec PCT was used and the sound was always presented. In another session, there was no sound. The instructions were the same as in Experiment 1 . This experiment intended to examine whether the subjects tended to equate the frequency of each percept, which might produce the pattern of the results in Experiment 1. (If subjects do not want to respond "streaming" all the time, they might report the bouncing percept whenever the percept is not clear.) With the presentation of the sound, all subjects $(N=4)$ reported the bouncing percept about $60 \%-80 \%$ of trials. Without the sound, they reported the streaming percept almost exclusively (about 98\%). These results of the control experiments show that the demand characteristics of the task did not alter the main result in the experiment.

(Manuscript received March 11, 1999;

revision accepted for publication March 24, 2000.)

\section{NOTICE}

\section{Change of Associate Editors}

We announce with regret the resignation of Associate Editor John Henderson. Professor Henderson has generously provided Perception \& Psychophysics with three years of his time and expertise.

At the same time, it is a pleasure to welcome Albrecht Inhoff of the University of Binghamton (SUNY) as a new associate editor. Professor Inhoff can be reached at inhoff@ binghamton.edu.

-Neil A. Macmillan

Editor 